\title{
METLIN Neutral Loss Database Enhances Similarity Analysis
}

\author{
Aries Aisporna, ${ }^{\dagger}$ H. Paul Benton, ${ }^{\dagger}$ Jean Marie Galano, ${ }^{\&}$ Martin Giera, ${ }^{\sharp}$ and Gary Siuzdak ${ }^{\dagger, \ddagger, *}$ \\ ${ }^{\dagger}$ Scripps Center for Metabolomics and Mass Spectrometry, The Scripps Research Institute, 10550 North \\ Torrey Pines Road, La Jolla, CA 92037 \\ \# Institut des Biomolécules Max Mousseron, UMR 5247 CNRS, ENSCM, Université de Montpellier, France \\ \&Leiden University Medical Center, Center for Proteomics and Metabolomics, Albinusdreef 2, 2333ZA Leiden, \\ Netherlands \\ ${ }^{\ddagger}$ Department of Chemistry, Molecular and Computational Biology, The Scripps Research Institute, 10550 \\ North Torrey Pines Road, La Jolla, CA 92037
}

*Corresponding author

Gary Siuzdak, PhD

Scripps Center for Metabolomics

The Scripps Research Institute, La Jolla, CA 92037

Tel.: 8587849425

Email: siuzdak@scripps.edu

Abstract: Tandem mass spectrometry $\left(\mathrm{MS}^{2}\right)$ data is an effective resource for the identification of known molecules and the putative identification of novel, previously uncharacterized molecules (unknowns). Yet, $\mathrm{MS}^{2}$ data alone is limited in characterizing structurally closely related molecules with different masses. Neutral loss data is key in retrieving this structural similarity. To facilitate unknown identification and complement METLIN's MS² fragment ion data for characterizing structurally related molecules, we have created the METLIN neutral loss database (https://metlinnl.scripps.edu).

Similarity analysis ${ }^{1-4}$ and molecular networking ${ }^{5,6}$ using tandem mass spectrometry $\left(\mathrm{MS}^{2}\right)$ data have become valuable approaches for identifying previously uncharacterized molecules (unknowns). ${ }^{1}$ Yet key structural information can be lost when relying solely on this fragment ion data, for example, the loss of a sulfate ion from two similar molecules of different masses will not result in fragment ion overlap. ${ }^{7}$ This is of significant practical relevance. A user who would try to identify an unknown based on a database similarity search would not succeed in obtaining structurally useful matches. However, retrieving this structurally useful information is possible by analyzing the differences between the molecular ion and the fragment ion, or better known as the neutral loss $(\Delta m / z)$. Neutral losses ${ }^{1,2}$ constitute a rich resource, and have already been widely used in proteomics, pharmacology, and metabolomics for over three decades. ${ }^{1,2,8-12}$ Yet, even though mass spectrometry-based neutral loss (NL) analysis has been extensively applied, with hundreds to 
thousands of papers on the topic, no comprehensive small molecule library of neutral loss data exists.

The new METLIN neutral loss database (METLIN-NL) has been created from METLIN's 850,000 MS² small molecule molecular standards database to facilitate neutral loss searching. The neutral loss data was derived across a broad range of standards representing hundreds of different chemical classes. ${ }^{3,13}$ METLIN's MS² data was converted to METLIN-NL spectra (e.g. Figure 1 asymmetric dimethylarginine (ADMA)) by calculating the differences between the precursor molecular ion and the fragment ions in the experimental $\mathrm{MS}^{2}$ mass spectra (Figure 1a). The neutral loss spectra

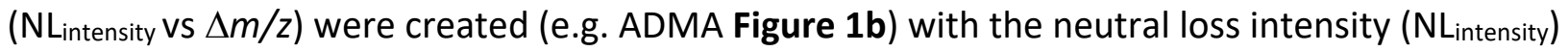
using the fragment ion intensities from each precursor/fragment generated neutral loss $(\Delta m / z)$. It should be noted that not all precursor to fragment peaks represent a true neutral loss between the precursor and fragment ions, and therefore some of the peaks in the NL spectra can also be considered (as recently described) hypothetical neutral losses.

METLIN-NL is a compilation NLintensity vs $\Delta m / z$ spectra generated from METLIN's eight distinct MS ${ }^{2}$ data sets ${ }^{3}$ created from 850,000 standards. This compilation is represented within METLIN-NL at four different collision energies and in both positive and negative ionization modes. The rationale behind providing multiple conditions is that $\mathrm{MS}^{2}$ collision energies have not been standardized and such broad acquisition parameters are required to represent the output across different instrument types. An additional rationale for the array of conditions is that different molecules can fragment differently depending on the collision energies thus METLIN provides a broad range of empirical data across its 850,000 standards. It is worth noting that all of METLIN's MS ${ }^{2}$ data is empirical data and has not been generated using predictive in silico-based approaches.

(A) Tandem Mass Spectra (ADMA)
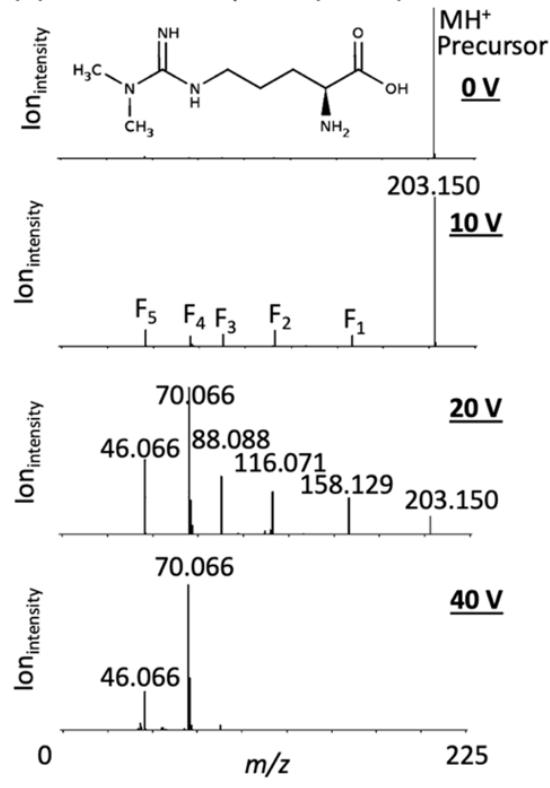

(B) Neutral Loss Mass Spectra (ADMA)
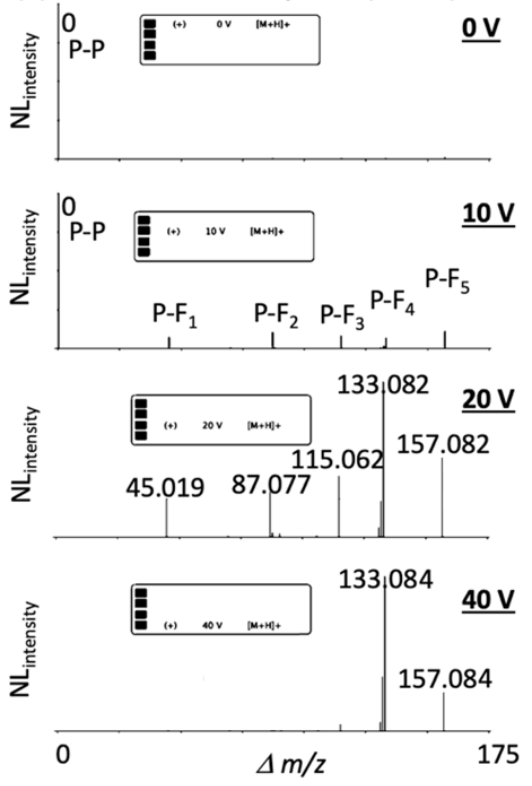

Figure 1. The METLIN-NL mass spectral database was derived from the METLIN MS ${ }^{2}$ data on over 850,000 molecular standards, and their respective fragment ions. (A) Asymmetric dimethylarginine (ADMA) and its representative METLIN tandem mass spectra at four different collision energies. (B) METLIN-NL spectra (NLintensity vs. $\triangle \mathrm{m} / \mathrm{z}$ ) of ADMA was generated by calculating the difference between the precursor and fragment ions with $N L_{\text {intensity based on the }}$ original fragment ion intensities. " $P$ " refers to precursor ion and " $F$ " refers to fragment ion. 
A secondary set of METLIN-NL data has also been accumulated based on precursor minus fragment ion transitions as well as all possible fragment to fragment ion transitions to provide a more comprehensive set of experimentally derived structural data. Unlike the original METLIN MS ${ }^{2}$ database, METLIN-NL represents a translation that more effectively enables the molecular annotation of unknown molecular entities since NL data is inherently corrected for molecular weight differences (Figures 2).

To test the utility of METLIN-NL we examined two different types of molecular structures, oxylipins and a pharmaceutical (statin) drug and its demethylated metabolite. Oxylipins ${ }^{14}$ represent a class of highly active lipid metabolites ubiquitous in humans and plants, and specifically, the phytoprostanes (PhytoPs) class of oxylipins resemble prostaglandin-like compounds that are found in seeds and vegetable oils derived from oxidative cyclization of $\alpha$-linolenic acid. Since PhytoPs are a class of highly structurally related oxylipins and are suspected to have additional unidentified analogs, ${ }^{14-16}$ we chose them to demonstrate the utility of METLIN-NL. Tandem MS and neutral loss data were recently generated on a set of PhytoPs, including the structural analogs 16- $\mathrm{B}_{1}$-PhytoP and 16-keto 16- $\mathrm{B}_{1}$-PhytoP (Figure 2). When trying to extrapolate/correlate the observed tandem MS spectra of the two PhytoPs, classic similarity searching was of very limited value providing only one overlapping ion, even though some fragments presented an expected two Dalton difference (Figure 2a). This exemplifies that two structurally very similar molecules can yield highly different $\mathrm{MS}^{2}$ spectra limiting similarity searching possibilities and thereby severely impacting the usefulness of this approach for the identification of chemically closely related substances. However neutral loss similarity analysis yielded multiple overlapping neutral losses (Figure $\mathbf{2 b}$ ). Further analysis of the tandem MS data as well as the molecular weight difference between the two molecules being 2 Daltons, were consistent with 16-keto 16-B1-PhytoP. This neutral loss data (unlike the $\mathrm{MS}^{2}$ data) helped to easily correlate the two molecules, and the distinguishing neutral losses and fragment ions exclusive to $16-$ keto $16-\mathrm{B}_{1}-\mathrm{Phyto} \mathrm{P}$ and $16-\mathrm{B}_{1}-\mathrm{Phy}$ to provides significant structural information.

The purpose of having a large database is to help reduce the need for speculation, and allow for the rapid identification of molecules. However, since many molecular structures are not represented in any database, similarity analyses offer an alternative in the preliminary characterization process. This process extends beyond naturally occurring molecules and can be applied just as readily to xenobiotics and other chemical entities. The second example in applying METLIN-NL is shown here for a non endogenous drug molecule and its metabolite.

The well known cholesterol-lowering statin drug rosuvastatin ${ }^{17}$ (trade name Crestor) and its active metabolite desmethyl rosuvastatin ${ }^{18}$ differ in mass by 14 Daltons (demethylation reaction) and the $\mathrm{MS}^{2}$ and neutral loss data (Figure $\mathbf{2 c} \boldsymbol{\&} \mathbf{2 d}$ ) of these two molecules have recently been acquired and populated within METLIN and METLIN-NL. As was observed with the oxylipins, tandem MS data was of limited utility when searching METLIN (Figure 2c), where 3 fragment ions were overlapping between the two molecules. However neutral loss matching/detection showed near complete overlap (Figure 2d). Further analysis of the tandem MS data as well as the molecular weight difference between the two molecules being 14 Daltons, were consistent with loss of a methyl group. For the rosuvastatin NL data, the overlap in the neutral loss data clearly dominated the comparative analyses, making similarity searching much more effective using neutral loss while the $\mathrm{MS}^{2}$ data provided complementary information that was informative for structural determination. Overall, the neutral loss data which was completely derived from the $\mathrm{MS}^{2}$ data, is more effective (than $\mathrm{MS}^{2}$ ) at showing similarity. 
(A) Tandem Mass Spectrum (-20V)

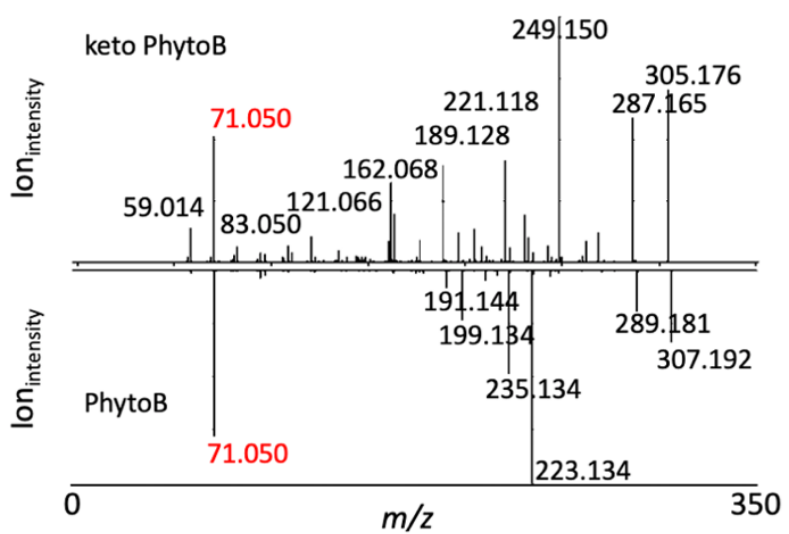

(C) Tandem Mass Spectra (+40 V)

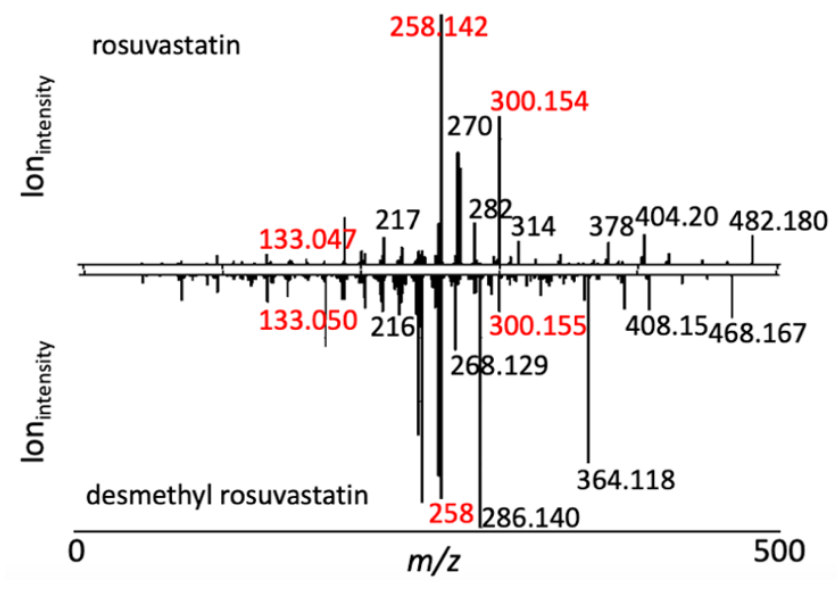

(B) Neutral Loss Mass Spectrum (-20V)

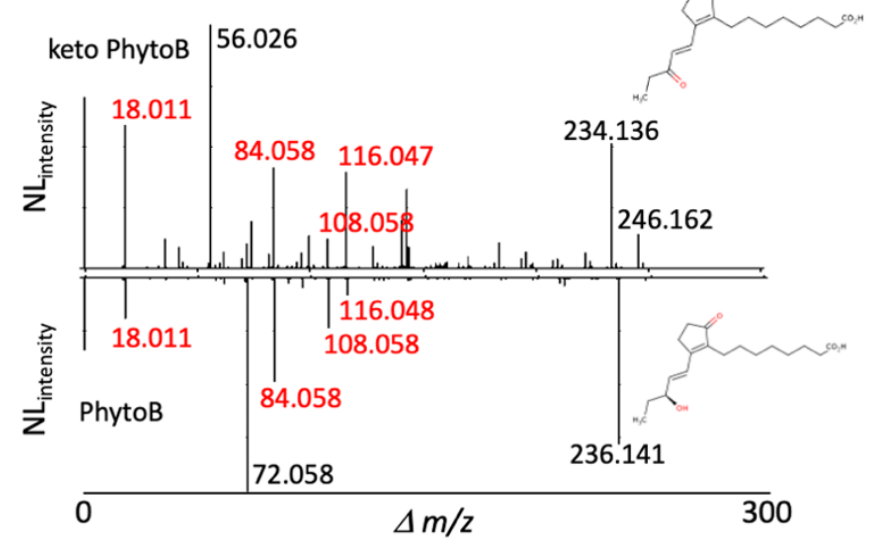

(D) Neutral Loss Mass Spectra (+40 V)

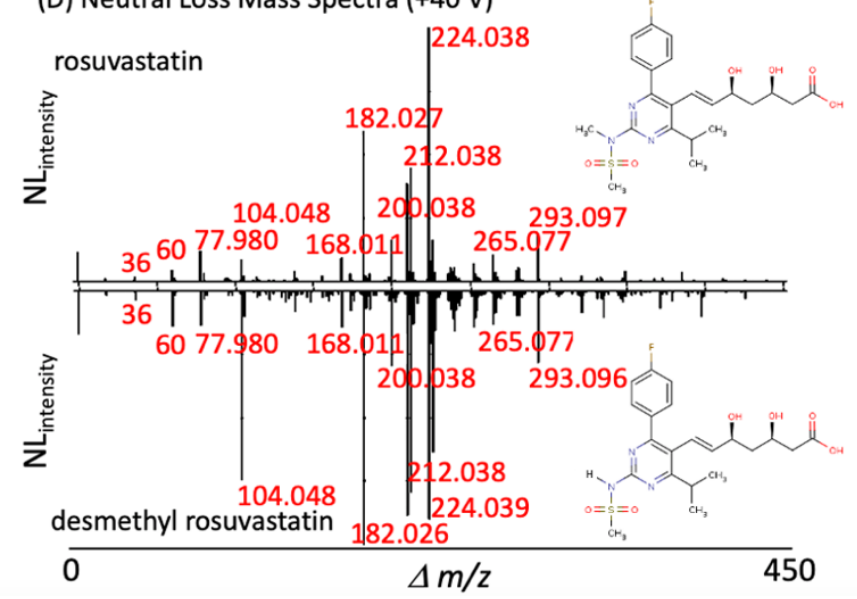

Figure 2. $M S^{2}$ and neutral loss data on two related oxylipins (16 keto 16- $B_{1}-$ PhytoP and 16-B 1 -PhytoP) and the statin drugs rosuvastatin and desmethyl rosuvastatin. (A) Oxylipin $\mathrm{MS}^{2}$ data show little overlap (in red) in contrast to the (B) neutral loss spectra with the high resolution neutral loss data facilitating similarity analysis with both providing complementary structural information. (C) $M^{2}$ and (D) neutral loss data on rosuvastatin and desmethyl rosuvastatin. $\mathrm{MS}^{2}$ data show few overlapping peaks (in red) while the neutral loss spectra provide near complete overlap. Interestingly, while the neutral losses help facilitate similarity, the $M S^{2}$ data provides more structural information on their structurally distinguishing features.

METLIN's molecular standards with systematically acquired experimental $\mathrm{MS}^{2}$ data across multiple collision energies, allows for the comprehensive generation and graphical user interface (beta) visualization (Figure 3) of neutral loss data. Fragment ion and neutral loss similarity analysis ${ }^{1}$ was originally developed to aid in the identification of novel molecules (unknowns) ${ }^{1}$ by using fragment ion and neutral loss data to help align an unknown molecule to compounds with similar fragmentation data within a database. However now, with a neutral loss database of small molecules via METLIN-NL, neutral loss similarity analysis can be more readily applied to a host of biological and chemical challenges.

Overall, METLIN-NL empirically derived data will enable new types of analyses facilitating more rapid identification of unknown compounds via both fragment ion and neutral loss similarity searching. ${ }^{2}$ Both biologists and chemists will be able applying METLIN-NL to the structure elucidation of unknowns derived from animals, ${ }^{19}$ plants, ${ }^{14,20}$ or microbiota ${ }^{21}$; and METLIN-NL can 
also be used as a resource for identifying unexpected synthetic chemical or enzymatically modified drug products (e.g. pharmaceuticals ${ }^{22}$ ) as it is populated with both biological and chemical entities. Given METLIN's extensive userbase, ${ }^{3}$ and the ubiquitous application of mass spectrometry-based neutral loss analysis (dating back three decades), METLIN-NL promises to have wide-ranging utility.

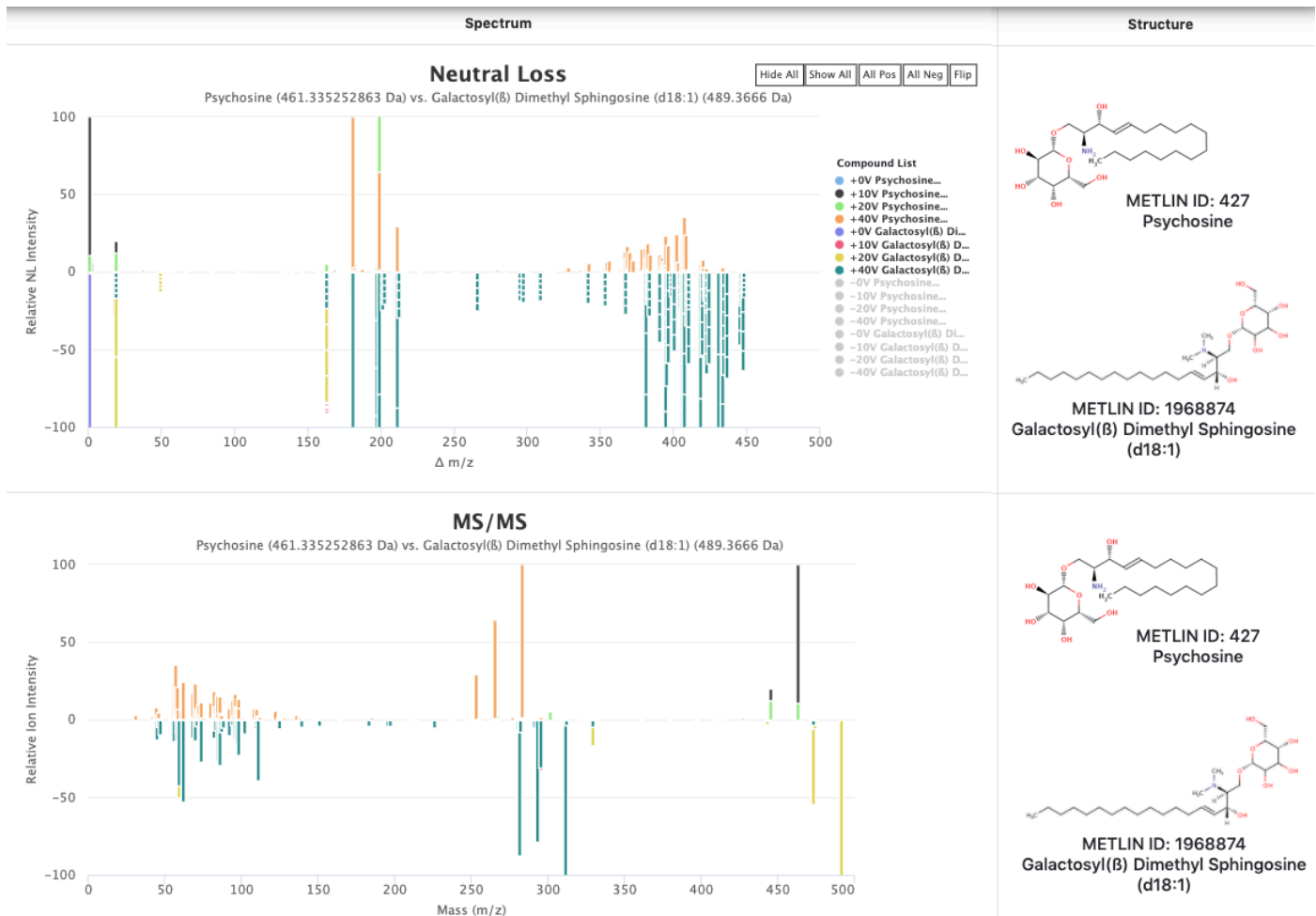

Figure 3. METLIN-NL is built on a Linux platform with this beta version of the graphical user interface (GUI) created using Highcharts, HTML, JQuery and PHP. The beta GUI allows for comparative analyses between different compounds including neutral loss data ( $N L_{\text {int }}$ vs $\Delta \mathrm{m} / \mathrm{z}$ ) as well as $M S / M S$ data (Frag int $_{\text {vs }} \mathrm{m} / \mathrm{z}$ ) in both positive and negative ionization modes and either at each individual collision energy, or a composite of multiple collision energies, as shown here for psychosine and gal dimethyl sphingosine. The Neutral Loss and MS/MS spectra are a composite of all the collision energies in positive ionization mode.

\section{Acknowledgements}

This research was partially funded by National Institutes of Health grants R35 GM130385 (G.S.), P30 MH062261 (G.S.), P01 DA026146 (G.S.), and U01 CA235493 (G.S.) and by Ecosystems and Networks Integrated with Genes and Molecular Assemblies (ENIGMA), a Scientific Focus Area Program at Lawrence Berkeley National Laboratory for the U.S. Department of Energy, Office of Science, Office of Biological and Environmental Research, under contract number DE-AC02-05CH11231 (G.S.).

\section{AUTHOR CONTRIBUTIONS}

A.A., H.P.B., J.M.G., M.G. and G.S. contributed to data collection, analysis, and manuscript writing. 


\section{References}

1. Benton, H.P., et al. Anal. Chem. 80, 6382-6389 (2008). 10.1021/ac800795f

2. Guijas, C. et al. Anal. Chem. 90, 3156-3164 (2018). 10.1021/acs.analchem.7b04424

3. Xue, J. et al. Nat. Methods 17, 953-954 (2020). 10.1038/s41592-020-0942-5

4. Tautenhahn, R. et al. Nat. Biotechnol. 30, 826-828 (2012). 10.1038/nbt.2348

5. Dapeng Li, D. et al. PNAS 112 (30) E4147-4155 (2015). 10.1073/pnas.1503106112

6. Watrous, J. et al. PNAS 109 (26)E1743-E1752 (2012). 10.1073/pnas.1203689109

7. Flasch, M. et al. ACS Chem. Bio. 15, 970-981 (2020). 10.1021/acschembio.9b01016

8. Martin, D.B. et al. Anal. Chem. 77, 4870-4882 (2005). 10.1021/ac050701k

9. Horai, H. et al. J. Mass Spectrometry 2010, 45(7) 703-714 (2010). 10.1002/jms.1777

10. Xing, S. et al. Anal. Chem. 92, 14476-14483 (2020). 10.1021/acs.analchem.0c02521

11. Heller, D.N. et al. Anal. Chem. 60, 2787-2791 (1988). 10.1021/ac00252a023

12. Schwudke, D. et al. Anal. Chem. 78, 585-595 (2006). 10.1021/ac051605m

13. Feunang, Y.D. et al. Journal of Cheminformatics 8, 61 (2016). 10.1186/s13321-016-0174-y

14. Galano, J.M. et al. Progress in Lipid Research, 68, 83-108 (2017). 10.1016/j.plipres.2017.09.004

15. Watrous, J.D. et al. Cell Chem. Biol., 26(3), 433-442 (2019). 10.1016/j.chembiol.2018.11.015

16. Young, R.S.E., et al. Cell Reports, 34(6) (2021). 10.1016/j.celrep.2021.108738

17. Fellström, B.C. et al. N. Engl. J. Med., 360, 1395-1407 (2009). 10.1056/nejmoa0810177

18. Martin, P.D. et al. Clin. Therapeutics 25(11) 2822-2835 (2003). 10.1016/S0149-2918(03)80336-3

19. Rosenberg, G. et al. Science 371(6527) 400-405 (2021). 10.1126/science.aba8026

20. Lipan, L. et al. J. Agric. Food Chem. 68(27) 7214-7225 (2020). 10.1021/acs.jafc.0c02268

21. Guo, H. et al. Science 370, 6516, (2020). 10.1126/science.aay9097

22. Giera, M. et al. Rapid Comm. Mass Spectrom. 24(10) 1439-1446 (2010). 10.1002/rcm.4534 